\title{
ANESTESIA ESPINAL CONTINUA EN CARDIOPATIAS SEVERAS PARA CIRUGÍAS DE FRACTURA DE CADERA. REVISIÓN DE CASOS. HOSPITAL MILITAR DE SANTIAGO - COMPLEJO HOSPITALARIO SAN JOSÉ
}

Lemus Paz de la Vega Roxana ${ }^{1}$, Alarcón Vaccarezza Patricia², Gundersen Kling, Hermann³ ${ }^{3}$ Salas Parada Andrés ${ }^{4}$,

Vozmediano Pérez Alejandro ${ }^{2}$

Médico Anestesiólogo. Instructor primero Universidad de Santiago. Hospital San José.

Médico Anestesiólogo. Instructor Universidad de los Andes. Hospital Militar de Santiago.

Residente 3 año. Anestesiología y Reanimación .Universidad de Santiago.

Residente 1 año. Anestesiología y Reanimación. Universidad de Santiago.

Introducción: La mayoría de los pacientes con fractura de cadera corresponden al grupo geriátrico cuya reserva fisiológica cardiovascular es limitada presentando además severas cardiopatías y otras comorbilidades no optimizables. Esto deriva en una elevada morbimortalidad por lo que el manejo perioperatorio y la elección de técnica anestésica es crucial. Objetivo General: Evaluar la efectividad de un protocolo de anestesia espinal continua ${ }^{1}$ (AEC) en pacientes portadores de cardiopatía severa con fractura de cadera sometidos a reducción y osteosíntesis de la misma.
Material y Métodos: Se revisaron las fichas clínicas de pacientes operados de fractura de cadera de marzo de 2014 a junio de 2016 portadores de cardiopatías severas y en los cuales se utilizó una técnica AEC protocolizada. El protocolo consiste en: realizar bloqueo regional femoral y obturador eco guiados previo a la AEC. Colocar catéter espinal y administrar bolos cada 20-30 min de 1 $\mathrm{ml}$ de solución de Bupivacaína $0,1 \%+$ Fentanilo $2,5 \mu \mathrm{g} / \mathrm{ml}$. Previo a retirar del catéter administrar $100 \mu \mathrm{g}$ de morfina. Monitorizar con ECG continuo, oxímetro de pulso y presión arterial invasi$\mathrm{va}^{2}$. No permitir disminución $>20 \%$ de presión

Tabla 1. Datos demográficos morbilidad cardiovascular y asociada

\begin{tabular}{|c|c|c|c|c|c|c|c|c|c|c|c|c|c|}
\hline Pac & Edad & Hta & DM & C. coro & ICC & ERC & $\mathrm{ACV}$ & DHC & $\begin{array}{l}\text { Ac } \\
\text { FA }\end{array}$ & $\mathbf{x}$ & NEO & $\mathbf{E a}^{\circ}$ & FEVI \\
\hline 1 & 95 & $\mathrm{x}$ & & $\mathrm{x}$ & $\mathrm{x}$ & & & $\mathrm{x}$ & & & & & $15 \%$ \\
\hline 2 & 89 & $\mathrm{x}$ & & & & & & & & & & $0,7 \mathrm{~cm}^{2}$ & $\mathrm{~N}$ \\
\hline 3 & 78 & $\mathrm{x}$ & $\mathrm{x}$ & $\mathrm{x}$ & & & $\mathrm{x}$ & & & & & & $30 \%$ \\
\hline 4 & 85 & $\mathrm{x}$ & & $\mathrm{x}$ & & $\mathrm{x}$ & & & & & $\mathrm{x}$ & & $\mathrm{N}$ \\
\hline 5 & 80 & $\mathrm{x}$ & $\mathrm{x}$ & $\mathrm{x}$ & & & & & & & & & $22 \%$ \\
\hline 6 & 92 & $\mathrm{x}$ & $\mathrm{x}$ & & & & & & & & & & $30 \%$ \\
\hline 7 & 86 & $\mathrm{x}$ & & & $\mathrm{x}$ & $\mathrm{x}$ & & $\mathrm{x}$ & $\mathrm{x}$ & & & & $40 \%$ \\
\hline 8 & 73 & $\mathrm{x}$ & & & $\mathrm{x}$ & & & & $\mathrm{x}$ & & & & $35 \%$ \\
\hline 9 & 93 & $\mathrm{x}$ & $\mathrm{x}$ & & & & & & & & & $0,8 \mathrm{~cm}^{2}$ & $\mathrm{~N}$ \\
\hline 10 & 82 & $\mathrm{x}$ & & & & & & & & & & & $30 \%$ \\
\hline 11 & 71 & $\mathrm{x}$ & $\mathrm{x}$ & & & $\mathrm{x}$ & & & $\mathrm{x}$ & & & & $30 \%$ \\
\hline
\end{tabular}

$\mathrm{C}=$ Cardiopatía; HTA $=$ Hipertensiva; Coro $=$ Coronaria $; \mathrm{Ea}^{\circ}=$ Estenosis aortica; $\mathrm{ICC}=$ Insuficiencia Cardiaca Congestiva; $\mathrm{DM}=$ Diabetes Mellitus; $\mathrm{ACV}=$ Accidente Cerebro Vascular; ACxFA = Fibrilación auricular; ERC

$=$ Enfermedad Renal Crónica; $\mathrm{DHC}=$ Daño Hepático Crónico; FEVI = fracción eyección ventrículo izquierdo; $\mathrm{N}=$ Normal. 
arterial media (PAM) basal en base a infusión de fenilefrina; oxígeno necesario para saturación $>$ $90 \%$; sedar con TCI propofol. El postoperatorio de todos los pacientes se realiza en UTI. Se obtuvieron los datos demográficos, patologías asociadas, exámenes, ecocardiografía, variables hemodinámicas intraoperatorias, número de bolos, complicaciones, tiempo quirúrgico y sobrevida a 6 meses.

Resultados: Durante el período, 11 pacientes ASA IV con cardiopatías severas fueron intervenidos por fractura de cadera con AEC; la edad promedio fue de 84 años, $81 \%$ sexo femenino, la capacidad funcional no fue evaluable en todos los pacientes. Las cardiopatías observadas fueron: 2 pacientes con estenosis aórtica $<1 \mathrm{~cm}^{2}$, 2 pacientes con cardiopatía coronaria inestable, 7 pacientes con antecedentes de insuficiencia cardíaca con fracción de eyección $<40 \%$ y con episodios de edema pulmonar asociado a cardiopatía coronaria. Requirieron en promedio 3,18 bolos, el tiempo de duración de cirugía fue 84 minutos con un sangrado promedio $222 \mathrm{ml}$; la dosis de Fenilefrina promedio fue de $0,31 \mathrm{ug} / \mathrm{kg} /$ min. Sólo 6 pacientes requirieron sedación a TCI
Propofol 0,325 ug/ml. Las técnicas quirúrgicas fueron: 9 casos con reducción y osteosíntesis y 2 casos con prótesis parcial. El promedio de estadía en UTI fue de 1,8 días y no se registraron complicaciones cardiovasculares ni mortalidad.

Conclusiones: La administración de anestésicos locales en pequeñas dosis efectivas y el manejo activo en la mantención de la PAM demostraron ser factibles y eficientes en no afectar la condición cardiovascular basal de este grupo demostrado por la ausencia de mortalidad y de complicaciones cardiovasculares perioperatorias.

\section{Referencias}

1. Hemodinamic profile of target controlled spinal anesthesia compared with two target controlled general anesthesia techiques in ederly patients with cardiac comorbilities. P. Biboulet $\mathrm{MD}$,et al. Regional Anesthesia and Pain Medicine. Vol 37, Nª, jul-agost 2012.

2. Lidco-based fluid management in patients undergoing hip fracture surgery under spinal anaesthesia: a randomized trial and systematic review. I.K. Moppett, et al. British Journal Anaesthesia 114(3):444-59(2015). 